\title{
Key elements in the statistical analysis of surveys
}

\author{
André Berchtold \\ Groupe de Recherche sur la Santé des Adolescents, Institut Universitaire de Médecine Sociale et Préventive, \\ Centre Hospitalier Universitaire Vaudois, Suisse \\ Institut de Mathématiques Appliquées, Université de Lausanne, Suisse
}

Submitted: 13 June 2006; Revised: 20 November, 4 December 2006; Accepted: 29 January 2007

This Hints \& Kinks paper summarizes the main points related to the statistical analysis of survey data. We emphasize first on the specificity of such data, then we compare two different statistical approaches. This paper should prove useful to researchers used to traditional statistical analysis, but without particular knowledge of the specificities of survey data. More generally, it can also serve as a reminder for all statisticians.

Most surveys are characterized by the complexity of the sampling design used to obtain data representative of the underlying population (Groves et al. 2004; Korn \& Graubard 1999). In many cases, the population is divided first in strata corresponding to clearly different groups of population, then a sample is taken from each strata. Moreover, samples are often composed of subjects sharing some important characteristics, and we speak then of clusters of observations. For instance, in a study concerned about the cannabis consumption by high school students in Switzerland, we could decide to take a sample of students in each of the 26 Swiss cantons, then to select several classes of students in each canton and to interview all students belonging to these classes (Fig. 1). In this example, the cantons are the strata and the classes are the primary sampling units. Since it is likely that students belonging to the same class share some important features and so are correlated in some sense, each class is a cluster of observations.

In addition to strata and clusters, a third element, sampling (or probability) weights, is essential in the design of surveys. Sampling weights are defined as the inverse of the probability for a subject to be included in a sample taken from the population. They generally take non-integer values (Tibaldi et al. 2003). These weights are used to rectify the sample e.g. in case of oversampling of a part of the population, or for nonresponse adjustment.

When analysing survey data, it is essential to take into account all the elements of the design. Otherwise, variances

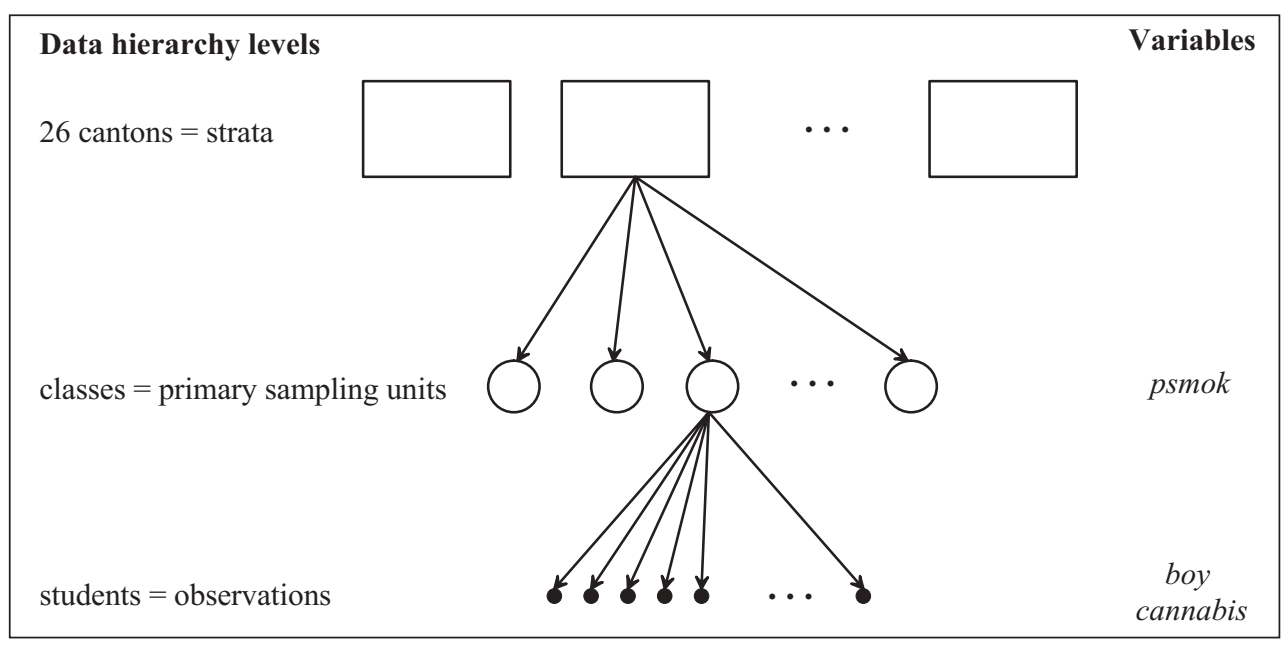

Figure 1 Three-level data hierarchy of the example survey 
computed from the sample will be too small, the consequences being confidence interval too narrows and a too high probability to reject the null hypothesis of tests (Crockett 2004). The inclusion of sampling weights and of strata and clusters into a statistical model are two different problems. Whatever the model, weights must always be included. For the researcher, the difficulty comes from the fact that many standard statistical packages such as SPSS or S-Plus do not allow for the use of sampling weights, at least in their standard version. The temptation is then high either to simply discard the weights or to treat the sampling weights as another type of weights allowed by the software, frequency weights in most cases. Such a practice must be absolutely banned, since results can be completely biased with not only errors on variance, but also on parameter point estimates. The only valid solution is to use software specialized in survey data analysis, such as Stata, the Complex Sample toolbox of SPSS, SAS/STAT, SUDAAN, or Wes Var (Brogan 2005).

Concerning strata and clusters, two elements have to be mentioned. First of all, in regard of a specific research question, it is not always necessary to take into account each element of the design. For instance, in the above example where Swiss cantons are strata, it could be possible that the effect of living in a particular canton does not change significantly the level of cannabis consumption. In that case, it is possible to simplify the design and not to include strata in the model. The effect of each element of the design upon a model can be evaluated through the deff measure (Kish 1965) defined as

$\operatorname{deff}=1+(n-1) \rho$

where $n$ is the average size of strata and $\rho$ is the intraclass correlation. The deff measure is interpreted as the expected ratio between a variance computed from data obtained through a complex design and the corresponding variance computed from data obtained through simple random sampling.

The statistical model itself used to explain the cannabis consumption can follow two completely different strategies. In the first one, a standard model such as a linear regression is defined for the dependent variable with all available explanatory factors, whatever at which level (students, classes, cantons) they apply. Since the design of the survey influences the model through the variance of each parameter, approximations of the true variances are obtained either by Taylor series linearization or by replication procedures such as the jacknife method. For instance, a model for the cannabis consumption of subject $i$ could be of the form

cannabis $_{i}=\beta_{0}+\beta_{1}$ boy $_{i}+\beta_{2}$ psmok $_{i}+e_{i}$ where cannabis is the number of days with cannabis consumption during the last 30 days, boy is an indicator variable identifying boys, psmok is the percentage of regular tobacco smokers in the class, and $e$ is a normally distributed error term. The cannabis and boy variables are computed at the subject level, when psmok is an average computed at the class level.

In this standard approach, the objective is to compute population estimations of parameters and the design of the survey is considered as a noise effect exogenous from the statistical model itself. By contrast, in a multilevel model (Hox 2002), the different levels of the data (subjects, classes, cantons) are constitutive elements of interest of the model. The value of the dependent variable is then seen as the combination of various influences coming from all levels of the data and the model is used to decompose the value into these effects. For instance, we could have the model

cannabis $_{i j k}=\gamma_{000}+\gamma_{100}$ boy $_{i j k}+\gamma_{010}$ psmok $_{j k}+\gamma_{110}$ boy $_{i j k}$ psmok $_{j k}$ $+e_{i j k}+u_{0 j k}+u_{1 j k}$ boy $_{i j k}+u_{00 k}+u_{10 k}$ boy $_{i j k}+u_{01 k}$ psmok $_{j k}+u_{11 k-}$ boy $_{i j k}$ psmok $_{j k}$

where $i, j$ and $k$ are respectively the indexes of subjects, classes, and cantons. Fixed parameters are named $\gamma$ and normally distributed error terms are named $e$ at the subject level and $u$ at the class and canton levels. The first line of the model groups all fixed effects, when the random part is on the second and third lines. The fixed part of the model tells that the number of cannabis consumption days is explained by variables boy and psmok, and by their interaction. The random part of the model indicates the magnitude of variation between subjects due to random differences coming from all three levels of the data hierarchy. The error terms $e_{i j k}$ specific to each subject, $u_{0 j k}+u_{1 j k} b_{y} y_{i j k}$ is the error attributable to random variations among classes, $u_{00 k}+u_{10 k} b y_{i j k}+u_{01 k}$ psmok $_{j k}+$ $u_{11 k} b o y_{i j k} p s m o k_{j k}$ and is the error attributable to random variation among cantons.

This model is complex, but it provides a very precise explanation of the dependent variable. Of course, it can be simplified by suppressing non-significant parameters. Moreover, if some elements of the design do not influence significantly the results, they can also be suppressed, leading to a more readable model. In our case, the analysis of the deff measure shows that the use of sampling weights and clusters is essential. Ignoring them would lead to an under-estimation of the variances by a factor 2. On the other hand, the use of strata does not add anything significant, so the model could be simplified by removing the canton level.

To summarize, a correct statistical analysis of survey data requires taking into account the whole design of the survey. Not 
doing so would result in incorrect variances, confidence intervals and p-values. The use of specialized softwares and /or specialized procedures such as multilevel models is then required. Table 1 compares the main characteristics of the two statistical approaches presented in the paper.
The writing of this paper would not have been possible without the support and pertinent comments of André Jeannin, Pierre-André Michaud and Joan-Carles Suris.

Table 1 Comparison of statistical approaches

\begin{tabular}{|c|c|}
\hline Standard approach & Multilevel approach \\
\hline The goal is to obtain population estimates of parameters. & $\begin{array}{l}\text { The goal is to understand the exact influence of explanatory factors } \\
\text { belonging to the different levels of the data hierarchy. }\end{array}$ \\
\hline $\begin{array}{l}\text { Easy to understand results, but modelling sometimes oversimplifying } \\
\text { the reality. }\end{array}$ & Modelling very close to the reality, but results complex to interpret. \\
\hline Sampling design exogeneous to the model. & Sampling design endogeneous to the model. \\
\hline \multicolumn{2}{|c|}{ Not taking into account the whole sampling design results in underestimated variances. } \\
\hline All variables are treated as if they belong to the same level. & $\begin{array}{l}\text { Each variable is introduced into the model at its own measurement } \\
\text { level. }\end{array}$ \\
\hline One global error term for the whole model. & $\begin{array}{l}\text { Several error terms indicating which part of the total variability is } \\
\text { attributable to each element of the model. }\end{array}$ \\
\hline \multicolumn{2}{|c|}{ The use of specialized statistical softwares is generally required. } \\
\hline Moderate computational time. & $\begin{array}{l}\text { Computational time can become prohibitive on complex models with } \\
\text { more than three levels and many factors. }\end{array}$ \\
\hline
\end{tabular}

\section{References}

Brogan DJ (2005). Sampling error estimation for survey data. In: Household Sample Surveys in Developing and Transition Countries, Chapter XXI. United Nations.

Crockett A (2004). Weighting the Social Surveys. UK Data Archive and Institute for Social and Economic Research. http://www.esds.ac.uk/ government/docs/weighting.pdf.

Groves RM, Fowler FJ, Couper MP, Lepkowski $J M$, Singer E, Tourangeau R (2004). Survey Methodology. Wiley Series in Survey methodology. New York: Wiley.
Hox JJ (2002). Multilevel Analysis: Techniques and Applications. London: Lawrence Erlbaum Associates.

Kish L (1965). Survey Sampling. New York: Wiley.

Korn EL \& Graubard BI (1999). Analysis of Health Surveys. Wiley Series in Survey methodology. New York: Wiley.

Tibaldi F, Bruckers L, Van Oyen H, Van der Heyden J, Molenbergh G (2003). Statistical software for calculating properly weighted estimates from Health Interview Survey Data. Social and Preventive Medicine 48: 269-71.
Address for correspondence

André Berchtold

Université de Lausanne

Institut de Mathématiques Appliquées

SSP, Anthropole

CH-1015 Lausanne

e-mail: Andre.Berchtold@unil.ch
To access this journal online:

http://www.birkhauser.ch/IJPH 